\title{
Perceptions, Expectations, Preferences, and
}

\section{Attitudes Toward Premature Ejaculation, Its}

\section{Diagnosis and Topical Treatment with Fortacin ${ }^{\mathrm{TM}}$ Spray: Results from an Expert Panel Discussion}

This article was published in the following Dove Press journal:

Research and Reports in Urology

\author{
Ferdinando Fusco (1D) \\ Massimiliano Creta $\left(\mathbb{D}^{2}\right.$ \\ Francesco Mangiapia ${ }^{2}$ \\ Lorenzo Cirigliano ${ }^{2}$ \\ Francesco Trama $\left.{ }^{2}\right)^{2}$ \\ Savio Domenico Pandolfo (D) $^{2}$ \\ Ciro Imbimbo ${ }^{2}$ \\ Nicola Longo ${ }^{2}$ \\ Vincenzo Mirone ${ }^{2}$ \\ 'Department of Urology, Luigi Vanvitelli \\ University of Naples, Naples, Italy; \\ ${ }^{2}$ Department of Neurosciences, \\ Reproductive Sciences and \\ Odontostomatology, University of \\ Naples Federico II, Naples, Italy
}

Introduction: Premature ejaculation (PE) represents the most prevalent male sexual issue. Before beginning treatment, it is essential to discuss the patient's expectations thoroughly.

Methods: Herein, we report the results of an expert panel discussion about perceptions, expectations, preferences, and attitudes towards PE, its diagnosis and treatment. The panel took place in October 2019 and involved 30 Italian urologists experienced in the management of male sexual dysfunction. It aimed at investigating physicians' points of view about selected aspects of PE management which emerged during the counselling of PE patients over the previous two years. Treatment-related questions were mainly focused on topical treatment with Fortacin ${ }^{\mathrm{TM}}$.

Results: Overall, $83.3 \%$ of those interviewed declared that most of their patients perceive $\mathrm{PE}$ as a bother rather than a disease. The percentage of urologists interviewed perceived that improved subjective control over ejaculation and prolonged intravaginal ejaculatory latency time (IELT) as the main benefit expected by the majority of their patients was $56.5 \%$ and $10 \%$, respectively. Eighty percent of urologists reported on-demand regimen as the dosage modality preferred by the majority of their patients and half of them reported the topical route to be the way of administration preferred in most cases. Moreover, $73.3 \%$ of urologists reported that adherence to treatment was higher in patients undergoing topical treatment. Finally, $80 \%$ of urologists perceived Fortacin to be efficacious in patients with acquired PE and $70 \%$ of them perceived its efficacy to be independent from IELT.

Discussion: Most patients perceive $\mathrm{PE}$ as a bother rather than a disease and mainly advocate an improved control over ejaculation. Fortacin is perceived as efficacious in patients with acquired PE, and independent from IELT.

Keywords: intravaginal ejaculatory latency time (IELT), premature ejaculation (PE), survey

\section{Introduction}

Premature ejaculation (PE) represents the most common male sexual issue with prevalence rates reaching $55 \%$ in men aged 50 to 59 years. ${ }^{1}$ It is characterized by: 1) ejaculation that always or nearly always occurs prior to or within about 1 minute of vaginal penetration from the first sexual experience (lifelong PE) or a clinically significant and bothersome reduction in latency time, often to about 3 minutes or less (acquired PE);2) an inability to delay ejaculation on all or nearly all vaginal penetrations; 3) negative personal consequences, such as distress, bother,
Correspondence: Massimiliano Creta Department of Neurosciences,

Reproductive Sciences and Odontostomatology, University of Naples Federico II, via Sergio Pansini 5, Naples 80I3I, Italy

Email max.creta@gmail.com 
frustration, and/or the avoidance of sexual intimacy. ${ }^{2} \mathrm{PE}$ involves a complex sensory pathway which can be targeted at two main sites: in the central nervous system by delaying in the transmission of ejaculation processing neural stimuli, and at the level of penile sensory receptors, by reducing penile sensitivity. ${ }^{3}$ Currently, pharmacotherapy represents the first-line treatment for lifelong PE. ${ }^{1}$ Oral treatment with selective serotonin re-uptake inhibitors (SSRIs) and/or Phosphodiesterase type 5 inhibitors (PDE5is) are strongly recommended. ${ }^{1}$ Topical anesthetic agents are considered a viable alternative to oral treatments. $^{1}$ For many years topical anesthetic agents have been prescribed as off-label treatments. More recently, Fortacin ${ }^{\mathrm{TM}}$ (Lidocaine/Prilocaine, Recordati) has been approved as the first officially topical therapy for primary PE. ${ }^{1}$ It was officially approved for use in the European Union in 2013 and finally launched in the UK in November 2016. ${ }^{4}$ Before beginning treatment, it is essential that an appropriate assessment of the PE patient is carried out and that there is a thorough discussion about expectations. ${ }^{5}$ Current physicians' and patients' perceptions, expectations, and preferences about PE, its diagnosis and treatment in everyday clinical practice are underinvestigated. Herein, we report the results from an expert panel discussion involving Italian urologists experienced in the management of male sexual dysfunctions aimed at describing their experience about perceptions, expectations, preferences, and attitudes towards PE, its diagnosis and treatment as emerged during the medical counselling of PE patients during the previous two years.

\section{Materials and Methods}

A semi-structured focus panel comprising 30 Italian urologists involved in the field of andrology for more than 10 years and experienced in the treatment of male sexual dysfunction took place at the University of Naples Federico II in October 2019. Participants were asked to complete a structured questionnaire involving eight closed-format questions about perceptions, expectations, preferences, and attitudes which emerged during the medical counselling of PE patients in the previous two years. Questions related to PE treatment were mainly focused on topical treatment with Fortacin (Table 1). An open collective discussion about the issues addressed in the questionnaire was then performed. The study was performed in accordance with the ethical standards laid down in the 1964 Declaration of Helsinki and its later amendments. This research did not require formal approval by the
Institutional Review Boards as it only describes the results of an expert panel discussion.

\section{Results}

The mean age of experts was 58.7 years (Standard Deviation: 6.9). Ten (33.3\%) worked in university hospitals, $12(40 \%)$ in private practice setting, and $8(26.6 \%)$ in community hospitals. The mean number of andrological patients seen in one year by the members of the panel was between 1400 and 1600, with about $30 \%$ of patients affected by PE. Results from the structured questionnaire are reported in Table 1.

\section{Discussion}

For the first time, we provided a contemporary picture, taken from Italian physicians' perspectives about perceptions, expectations, preferences, and attitudes toward some relevant and controversial issues about PE, its diagnosis and treatment. One of the most relevant findings we reportis the perception of PE by most patients as a bother rather than a disease. This observation has relevant pathogenetic and clinical implications and is in line with some published theories that do not consider PE as a true disease. ${ }^{6}$

From a pathogenetic point of view, the etiology of $\mathrm{PE}$ is not fully understood and is likely multifactorial. Although PE can be found in patients with organic diseases, in most cases psycho-relational factors are the main or unique determinants. ${ }^{6,7}$ Evolutionary theories further support the concept of PE as a bother rather than a disease. For hominids, a faster ejaculation was a key factor to increase the chances of fertilization, thus preserving the genome. ${ }^{7}$ When the human was in a stable relationship the frequency of sexual intercourse increased and the chances of fertilization improved. In the modern era, the control of ejaculation is perceived as an important feature of pleasure and couple's sexual health. ${ }^{7}$

From a clinical point of view, the prevalent subjective connotation of PE has potential diagnostic and therapeutic implications that involve both physicians' and patients' decisions.

Most of the experts involved in the panel discussion declared that they mainly adopted a simplified diagnostic approach that favors history and physical examination over patient reported outcome-based (PRO) questionnaires, stopwatch-measured intravaginal ejaculatory latency time (IELT), laboratory, and neuro-physiological tests. This attitude of diagnosing PE mainly on the basis of patients' complaints further reinforced, from a physician's 
Table I Results from the Structured Questionnaire

\begin{tabular}{|c|c|c|c|}
\hline \multicolumn{2}{|c|}{ Question } & \multirow{2}{*}{$\begin{array}{l}\text { Answer } \\
\text { As a disease } \\
\text { As a bother }\end{array}$} & \multirow{2}{*}{$\begin{array}{l}\mathbf{N}(\%) \\
5(16.6) \\
25(83.3)\end{array}$} \\
\hline 1 & How PE is perceived by the majority of your patients? & & \\
\hline 2 & $\begin{array}{l}\text { How frequently do you perform an extensive diagnostic work-up } \\
\text { including PROs-based questionnaires, stopwatch-measured IELT, } \\
\text { laboratory and neuro-physiological tests in addition to history and } \\
\text { physical examination in your patients with PE? }\end{array}$ & $\begin{array}{l}\text { Always } \\
\text { Sometimes } \\
\text { Never }\end{array}$ & $\begin{array}{l}3(10.0) \\
18(60.0) \\
9(30.0)\end{array}$ \\
\hline 3 & $\begin{array}{l}\text { What is the main benefit expected by the majority of your patients from } \\
\text { pharmacological therapy of PE? }\end{array}$ & $\begin{array}{l}\text { Improved control over ejaculation } \\
\text { Improved IELT } \\
\text { Both }\end{array}$ & $\begin{array}{l}17(56.5) \\
3(10.0) \\
10(33.3)\end{array}$ \\
\hline 4 & $\begin{array}{l}\text { What dosage regimen is preferred by the majority of your patients with } \\
\text { PE? }\end{array}$ & $\begin{array}{l}\text { Daily } \\
\text { On-demand } \\
\text { Both }\end{array}$ & $\begin{array}{l}4(13.3) \\
24(80.0) \\
2(6.6)\end{array}$ \\
\hline 5 & $\begin{array}{l}\text { What route of administration is preferred by the majority of your } \\
\text { patients with PE undergoing medical therapy? }\end{array}$ & $\begin{array}{l}\text { Oral } \\
\text { Topical } \\
\text { Both }\end{array}$ & $\begin{array}{l}12(40.0) \\
15(50.0) \\
3(10.0)\end{array}$ \\
\hline 6 & Is Fortacin ${ }^{\mathrm{TM}}$ efficacious in patients with acquired PE? & $\begin{array}{l}\text { Yes } \\
\text { No }\end{array}$ & $\begin{array}{l}24(80.0) \\
6(20.0)\end{array}$ \\
\hline 7 & Is the efficacy of Fortacin influenced by IELT? & $\begin{array}{l}\text { Yes, it is higher in patients with IELT< Imin } \\
\text { Yes, it is higher in patients with IELT>I min } \\
\text { No, it is independent from IELT }\end{array}$ & $\begin{array}{l}4(13.3) \\
5(16.6) \\
21(70.0)\end{array}$ \\
\hline 8 & $\begin{array}{l}\text { The adherence to medical therapy, in the majority of your patients with } \\
\mathrm{PE} \text { is higher for: }\end{array}$ & $\begin{array}{l}\text { Oral therapy } \\
\text { Topical therapy } \\
\text { Is not influenced by the route of } \\
\text { administration }\end{array}$ & $\begin{array}{l}4(13.3) \\
22(73.3) \\
4(13.3)\end{array}$ \\
\hline
\end{tabular}

Abbreviations: IELT, intravaginal ejaculatory latency times; PE, premature ejaculation; PROs, patient reported outcomes.

point of view, the concept of PE as a subjective bother, and is in line with results from previously published surveys, and is supported by current European Association of Urology Guidelines. ${ }^{1,4,8}$ Of note, although several questionnaires have been proposed, there is a low correlation between a diagnosis provided by questionnaires and a selfreported diagnosis and their role is optional in everyday clinical practice. ${ }^{1}$

A hierarchical relationship between the outcomes of $\mathrm{PE}$ treatment has been hypothesized. IELT and the perceived control over ejaculation have been reported to affect patients' satisfaction with sexual intercourse and their distress related to PE. These outcomes, in turn, influence interpersonal relationship between patients and their partners. ${ }^{9}$

From a therapeutic point of view, patients' expectations are mainly focused on improved subjective control over ejaculation rather than on objective elongation of IELT. PE is a multidimensional issue encompassing several components, including not only IELT but also subjective parameters such as "control", "satisfaction," and "distress." 10 Waldinger et al investigated IELT in a large group of healthy heterosexual males with neither ejaculation difficulties nor co-morbidities and who were in stable monogamous relationships. ${ }^{11}$ The authors found that the IELT distribution had a median duration of $5.4 \mathrm{~min}$ and a wide range of variability $(0.55-44.1 \mathrm{~min})^{7,11}$ Consequently, there is a significant overlap between men with and without PE in terms of IELT. On the other hand, it has been demonstrated that perceived control over ejaculation is central to beneficial treatment in men with PE. ${ }^{12}$ In their study, Patrick et al failed to demonstrate a significant direct effect of IELT on ejaculation-related personal distress or satisfaction with sexual intercourse. ${ }^{13}$ On the contrary, perceived control over ejaculation showed a significant direct effect on both ejaculation-related personal distress and satisfaction with sexual intercourse, which each showed direct effects on interpersonal difficulty related to ejaculation. ${ }^{13}$ 
With expanding treatment options, physicians now have the opportunity to discuss different treatment regimens and routes of administrations with their patients.

The majority of experts involved in this study's panel declared that most of their patients prefer the on-demand regimen over the daily one. Patients' preference for PE treatments regimens represents a matter of debate. In 2007, Waldinger et al investigated preferences in taking either continuous or on-demand drugs against PE. ${ }^{14}$ The study group consisted of a consecutive and representative group of men with lifelong PE aged between 18 and 65 years. The majority of these men $(81 \%)$ preferred to take a daily drug. ${ }^{14}$ Only $16 \%$ preferred the on-demand oral drug treatment, and only $3.4 \%$ preferred the on-demand use of topical anesthetic creams. ${ }^{14}$ Sixty percent of subjects who preferred the daily drug treatment as a first choice reported that this treatment modality might guarantee better spontaneity. ${ }^{14}$ On the other hand, about $60 \%$ of the men who preferred on-demand strategies as their first choice reported to be afraid of side effects induced by the daily drug. ${ }^{14}$ Hellstrom and Sharlip argued that the on-demand treatment strategy would be "ideal" to treat PE and that this treatment regimen would not interfere with the spontaneity of sexual performance. ${ }^{15-17}$ The expert involved in the present panel discussion declared, in the discussion session, that most patients perceive a daily regimen as an overtreatment with an unacceptable risk of side effects for a condition that is perceived as a bother.

With regard to the route of administration, most experts declared that their patients prefer the topical one. The use of topical anesthetics represents the oldest form of pharmacotherapy in PE and dates back to $1943 .{ }^{5}$ These agents are currently considered a viable alternative to oral treatments. ${ }^{1}$ These agents can be applied directly to the glans penis in order to produce some degree of penile desensitization and are directed to the hypersensitivity aspect of $\mathrm{PE}^{3}$ Advantages of topical treatments include on-demand dosing with minimal systemic effects. Indeed, as emerged from the panel discussion, the better toxicity profile associated with topical agents is perceived as more adequate to control a subjective symptom. The field of topical anesthetics for PE has evolved in recent years. First generation topical anesthetics had dosing difficulties with the potential of either over or under-dosing, with subsequent risk of penile hypoesthesia, erectile dysfunction and/or transvaginal transmission resulting in vaginal numbness and anorgasmia or lack of efficacy, respectively. ${ }^{3}$ Their ease of application and tolerability was further limited by the presence of a number of excipients in the formulation of the majority of creams and topical products causing difficulties in applying the correct measured dose. ${ }^{3,18}$ In addition, difficulties in applying the cream were reported. ${ }^{3,19,20}$ The recently approved Fortacin is a metered-dose aerosol spray and contains purely base forms of the local anesthetics lidocaine and prilocaine with no excipients, except the spray propellant. ${ }^{4}$ This formulation has been reported to optimize the penetration through the glans surface leading to a rapid reduction in penile sensitivity (within 5 minutes) without adversely affecting the sensation of ejaculation. Clinical trials, have shown significant benefits for both patients and their female partners in ejaculatory latency, control, and sexual satisfaction. $^{3}$

Men with acquired PE should receive etiology-specific treatment alone or in combination with $\mathrm{PE}$ pharmacotherapy. ${ }^{21}$ Although Fortacin has been formally approved as the first officially topical therapy for primary PE, most of the experts involved in this study's panel discussion consider it also efficacious in patients with acquired PE. Similarly, dapoxetine has been reported to be comparably effective both in men with lifelong and acquired PE. ${ }^{22}$ Although lifelong and acquired PE are characterized by different mean IELTs, this parameter should not be applied in the most absolute sense to differentiate the two conditions, that are rather characterized by a different pathophysiology. Indeed, about $10 \%$ of men seeking treatment for lifelong PE have IELTs of 1-2 minutes. ${ }^{23}$ The efficacy of Fortacin is perceived to be independent from IELT thus representing a valid therapeutic strategy across all PE severities. Other topical treatment options for PE include severance secret cream (SS-Cream), resiniferatoxin, and 4 treatment options that patients can purchase over the counter: K-Y Duration Spray for Men, Pre-boost, Promescent, and STUD-100. ${ }^{24}$ SS-Cream is a topical agent with local anesthetic and vasoactive effect derived from the following natural products: ginsenoside, eugenol, bufosterioid, and methyl eugenol. ${ }^{24}$ This treatment option is currently not available in Italy. Resiniferatoxin has a similar composition to capsaicin and is extracted from cactus plants. It has been reported to decrease the excitatory and sensitivity of afferent nerves. However, further studies are required to evaluate the effectiveness of this treatment option. Finally, treatment options that patients can purchase over the counter are not currently supported by controlled studies indicating their efficacy or safety. ${ }^{24}$ Adherence to PE treatment represents a controversial issue. In their study, Mondaini et al reported drop-out rates with dapoxetine of $26 \%, 42.7 \%, 18.7 \%$, and $2 \%$ after 1 , 
3,6 , and 12 months, respectively. ${ }^{17,25}$ Drop-out rates of about $90 \%$ within 1 year have been reported with dapoxetine in real-world clinical practice. ${ }^{5,26-28}$ Reasons given includ: not wanting to take an antidepressant, treatment effects below expectations, and cost. ${ }^{21-25}$ To date, there are no direct comparisons in terms of treatment adherence between oral and topical treatments for PE. Urologists involved in this study's discussion panel reported that, based on their experience, adherence to therapy was higher for topical therapy. In the open discussion it was reported that reasons for higher adherence to topical treatments were better safety profile and perception of topical agents as simple "aid" and not as a "drug." However, a careful evaluation of adherence to EP treatments is challenging. Indeed, drop-out rates do not necessarily imply a lack of adherence, and some subjects may simply interrupt the treatment due to improvement in the issue.

Results from this study's expert panel discussion point out the subjective connotation of $\mathrm{PE}$, the relevance of strategies aimed at improving mainly the control over ejaculation rather than objective parameters, as well as the high preference for on-demand treatments with low toxicity profile. Fortacin potentially meets most of these requirements and is perceived by most of the urologists interviewed to be efficacious not only in patients with lifelong PE but even in patients with acquired PE and independent from IELT. However, further studied are needed to provide adequate levels of evidences to identify the right positioning of this agent into the treatment algorithm of $\mathrm{PE}$ and its potential role as a first-line treatment for both primary and acquired PE.

Patient surveys are needed to confirm these preliminary data about PE perception and treatment preferences, and might diverge from the present report of urologists involved in our panel discussion. Similarly, well designed studied are needed to investigate the efficacy of Fortacin in patients with acquired $\mathrm{PE}$ and to evaluate the long-term adherence to this treatment compared to other options. Finally, additional relevant outcomes including patients' distress and satisfaction, partners' satisfaction and opinions about PE and its treatment deserve further investigation.

\section{Conclusion}

Based on experts' opinion, most patients perceive PE as a bother rather than a disease and mainly advocate an improved control over ejaculation rather a elongation of IELT. Ondemand treatment options and the topical route of administration are preferred. Most of the physicians interviewed declared that Fortacin is efficacious in patients with acquired $\mathrm{PE}$ and its efficacy is independent from IELT. However, this expert panel discussion should be considered as explorative and further studies are needed to provide adequate levels of evidence about the issue addressed and to identify the right positioning of Fortacin into the treatment algorithm of PE.

\section{Disclosure}

Vincenzo Mirone and Ferdinando Fusco are speakers for Recordati. Massimiliano Creta is a speaker and medical writer for Recordati. Ferdinando Fusco reports grants, personal fees from Recordati, during the conduct of the study. Massimiliano Creta reports grants, personal fees from Recordati SPA, personal fees, non-financial support from IBSA, during the conduct of the study. The authors report no other conflicts of interest in this work.

\section{References}

1. Hatzimouratidis K, Giuliano F, Moncada I, et al. EAU guidelines on male sexual dysfunction: erectile dysfunction and premature ejaculation; 2016. Available from: http://uroweb.org/guideline/male-sexual dysfunction/. Accessed April 13, 2017.

2. McMahon CG, Althof SE, Waldinger MD, et al. An evidence-based definition of lifelong premature ejaculation: report of the International Society for Sexual Medicine (ISSM) Ad Hoc Committee for the definition of premature ejaculation. J Sex Med. 2008;5:1590-1606. doi:10.1111/j.1743-6109.2008.00901.x

3. Porst H, Burri A. Fortacin ${ }^{\mathrm{TM}}$ Spray for the treatment of premature ejaculation. Urologia J. 2017;84(2_suppl):1-10. doi:10.5301/uj.5000275

4. Shindel A, Nelson C, Brandes S. Urologist practice patterns in the management of premature ejaculation: a nationwide survey. $J$ Sex Med. 2008;5:199-205. doi:10.1111/j.1743-6109.2007.00638.x

5. Porst H, Burri A. Novel treatment for premature ejaculation in the light of currently used therapies: a review. Sex Med Rev. 2019;7 (1):129-140. doi:10.1016/j.sxmr.2018.05.001

6. Puppo V, Puppo G. Comprehensive review of the anatomy and physiology of male ejaculation: premature ejaculation is not a disease. Clin Anat. 2016;29(1):111-119. doi:10.1002/ca.22655

7. Palmieri A, Imbimbo C, Longo N, et al. Ejaculatory abstinence influences intravaginal ejaculatory latency time: results from a prospective randomized trial. Urol Int. 2012;88(4):459-462. doi:10.1159/000337057

8. Seo DH, Jeh SU, Choi SM, et al. Diagnosis and treatment of premature ejaculation by urologists in South Korea. World J Men's Health. 2016;34(3):217-223. doi:10.5534/wjmh.2016.34.3.217

9. Revicki D, Howard K, Hanlon J, Mannix S, Greene A, Rothman M. Characterizing the burden of premature ejaculation from a patient and partner perspective: a multi-country qualitative analysis. Health Qual Life Outcomes. 2008;6:33. doi:10.1186/1477-7525-6-33

10. Broderick GA. Premature ejaculation: on defining and quantifying a common male sexual dysfunction. J Sex Med. 2006;3 Suppl 4:295302. doi:10.1111/j.1743-6109.2006.00304.x

11. Waldinger MD, Quinn P, Dilleen M, Mundayat R, Schweitzer DH, Boolell M. A multinational population survey of intravaginal ejaculation latency time. J Sex Med. 2005;2:492-497. doi:10.1111/j.17436109.2005.00070.x

12. Shabsigh R, Patrick DL, Rowland DL, Bull SA, Tesfaye F, Rothman M. Perceived control over ejaculation is central to treatment benefit in men with premature ejaculation: results from Phase III trials with dapoxetine. BJU Int. 2008;102(7):824-828. doi:10.1111/j.1464-41 0X.2008.07845.x 
13. Patrick DL, Rowland D, Rothman M. Interrelationships among measures of premature ejaculation: the central role of perceived control. $J$ Sex Med. 2007;4(3):780-788. doi:10.1111/j.1743-6109.2007.00464.x

14. Waldinger MD, Zwinderman AH, Olivier B, Schweitzer DH. The majority of men with lifelong premature ejaculation prefer daily drug treatment: an observation study in a consecutive group of Dutch men. J Sex Med. 2007;4(4 Pt 1):1028-1037. doi:10.1111/j.1743-6109.20 07.00528.x

15. Hellstrom WJ. Current and future pharmacotherapies of premature ejaculation. J Sex Med. 2006;3(suppl 4):332-341. doi:10.1111/j.17436109.2006.00309.x

16. Hellstrom WJ, Heintz JW. Treatment of premature ejaculation: new drugs and treatment strategies. Curr Urol Rep. 2006;7:473-478. doi:10.1007/s11934-006-0057-2

17. Sharlip I. Diagnosis and treatment of premature ejaculation: the physician's perspective. J Sex Med. 2005;2(suppl 2):103-109. doi:10.1111/j.1743-6109.2005.20370.x

18. Morales A, Barada J, Wyllie MG. A review of the current status of topical treatments for premature ejaculation. BJU Int. 2007;100 (3):493-501. doi:10.1111/j.1464-410X.2007.07051.x

19. Atikeler MK, Gecit I, Senol FA. Optimum usage of prilocaine-lidocaine cream in premature ejaculation. Andrologia. 2002;34(6):356359. doi:10.1046/j.1439-0272.2002.00511.x

20. Busato W, Galindo CC. Topical anaesthetic use for treating premature ejaculation: a double-blind, randomized, placebo-controlled study. $B J U$ Int. 2004;93(7):1018-1021. doi:10.1111/j.1464-410X.2003.04773.x

21. McMahon CG. Emerging and investigational drugs for premature ejaculation. Transl Androl Urol. 2016;5(4):487-501. doi:10.21037/ tau.2016.04.02
22. Porst H, McMahon CG, Althof SE, et al. Baseline characteristics and treatment outcomes for men with acquired or lifelong premature ejaculation with mild or no erectile dysfunction: integrated analyses of two Phase 3 dapoxetine trials. J Sex Med. 2010;7(6):2231-2242. doi:10.1111/j.1743-6109.2010.01820.x

23. McMahon CG, Jannini EA, Serefoglu EC, Hellstrom WJ. The pathophysiology of acquired premature ejaculation. Transl Androl Urol. 2016;5(4):434-449. doi:10.21037/tau.2016.07.06

24. Butcher MJ, Zubert T, Christiansen K, Carranza A, Pawlicki P, Seibel S. Topical agents for premature ejaculation: a review. Sex Med Rev. 2020;8(1):92-99. doi:10.1016/j.sxmr.2019.03.003

25. Mondaini N, Fusco F, Cai T, et al. Dapoxetine treatment in patients with lifelong premature ejaculation: the reasons of a "Waterloo". Urology. 2013;82:620-624. doi:10.1016/j.urology.2013.05.018

26. McMahon CG, Althof SE, Kaufman JM, et al. Efficacy and safety of dapoxetine for the treatment of premature ejaculation: integrated analysis of results from five phase 3 trials. J Sex Med. 2011;8:524539. doi:10.1111/j.1743-6109.2010.02097.x

27. Park HJ, Park NC, Kim TN, et al. Discontinuation of dapoxetine treatment in patients with premature ejaculation: a 2-year prospective observational study. J Sex Med. 2017;5(2):e99- e105. doi:10.1016/j. esxm.2017.02.003

28. Jern P, Johansson A, Piha J, et al. Antidepressant treatment of premature ejaculation: discontinuation rates and prevalence of side effects for dapoxetine and paroxetine in a naturalistic setting. Int $J$ Impot Res. 2015;27(2):75-80. doi:10.1038/ijir.2014.37
Research and Reports in Urology

\section{Publish your work in this journal}

Research and Reports in Urology is an international, peer-reviewed, open access journal publishing original research, reports, editorials, reviews and commentaries on all aspects of adult and pediatric urology in the clinic and laboratory including the following topics: Pathology, pathophysiology of urological disease; Investigation and treatment of urological disease; Pharmacology of drugs used for the treatment of urological disease. The manuscript management system is completely online and includes a very quick and fair peer-review system, which is all easy to use. Visit http://www.dovepress.com/ testimonials.php to read real quotes from published authors. 